\title{
AN APPROXIMATE SOLUTION OF SOME VOLTERRA INTEGRAL EQUATION WITH A SMOOTH KERNEL
}

Abstract. The behaviour near the origin of nontrivial solutions to integral Volterra equations with a power nonlinearity is studied. Estimates of nontrivial solutions are given and some numerical examples are considered.

1. Introduction. We consider a nonlinear Volterra integral equation of the type

$$
u(x)=\int_{0}^{x} k(x-s) g(u(s)) d s, \quad x \in[0, \delta], \delta>0,
$$

where the kernel $k$ is nonnegative and the nonlinearity $g$ is concave and increasing with $g(0)=0$. This type of equation appears in many applications, like nonlinear diffusion problems, studies of the shape of the pendant liquid drop or shock wave propagation. The equation (1) always has the trivial solution $u \equiv 0$, but more interesting are nontrivial ones, which are continuous functions $u$ such that $u(0)=0$ and $u(x)>0$ for $x>0$. Some results on the existence of such nontrivial solutions can be found e.g. in $[4,6,7]$. As proven in [1], if a nontrivial solution exists then it is unique.

Since in modelling phenomena mentioned above the function $g(u)$ behaves like $u^{p}, p \in(0,1)$, as $u \rightarrow 0^{+}$(see [7]), in this paper we study estimates of nontrivial solutions to the equation

$$
u(x)=\int_{0}^{x} k(x-s)[u(s)]^{p} d s, \quad x \in[0, \delta], p \in(0,1) .
$$

We give a priori estimates of such solutions near the origin and determine the interval of their validity. Since in the case $k(x)=x^{\lambda-1}(\lambda>0)$ some

2000 Mathematics Subject Classification: 45D05, 45G10, 45L05, 65R20.

Key words and phrases: nonlinear Volterra integral equation, estimates of nontrivial solutions. 
estimates were already considered in [3], we concentrate on kernels which are very smooth near the origin, like $k(x)=x^{-\lambda-1} \exp \left(-1 / x^{\lambda}\right)(\lambda>1)$. In such cases, the construction of a priori estimates is difficult and in effect, numerical handling of such equations is cumbersome.

2. Definitions and auxiliary lemmas. We consider equation (2) assuming that

$k:[0,+\infty) \rightarrow[0,+\infty)$ is a nondecreasing, continuous function such that $k(0)=0$ and $k(x)>0$ for $x>0$.

Let

$$
K(x)=\int_{0}^{x} k(s) d s \quad \text { for } 0 \leq x<+\infty .
$$

Then the function $K$ is strictly increasing. Let

$$
K_{\mathrm{inf}}=\lim _{x \rightarrow+\infty} K(x) .
$$

Without loss of generality we assume further that

$$
K_{\text {inf }} \geq 1 .
$$

The inverse function $K^{-1}(z)$ is defined for $0 \leq z<K_{\text {inf }}$. Throughout the paper, we fix parameters $p, q$ such that $0<p<q<1$ and denote by $\varepsilon \in(0,1)$ a small generic constant which is allowed to change its value from paragraph to paragraph.

One of the known necessary and sufficient conditions for the existence of a nontrivial solution to (2) is given in the following theorem [5]:

THEOREM 1. Equation (2) has a nontrivial solution if and only if

$$
I(x)<\infty
$$

for all sufficiently small $x>0$, where

$$
I(x)=\int_{0}^{x} K^{-1}(s) \frac{d s}{s(-\ln s)} .
$$

From now on we consider only kernels $k$ satisfying conditions (3) and (4). Then we note that we have $I(x)<+\infty$ for $0 \leq x<1$ and $I(1)=+\infty$, and moreover

$$
\left(-\ln \frac{\ln b}{\ln a}\right) K^{-1}(a) \leq \int_{a}^{b} K^{-1} \frac{d s}{s(-\ln s)} \leq\left(-\ln \frac{\ln b}{\ln a}\right) K^{-1}(b),
$$

whenever $0<a<b<1$. 
We define

$$
\begin{array}{ll}
\bar{S}(x)=\sum_{n=0}^{\infty} K^{-1}\left(x^{(1-q)(q / p)^{n}}\right), & 0 \leq x<1, \\
\underline{S}(x)=\sum_{n=0}^{\infty} K^{-1}\left((1-\varepsilon) x^{(1-p)(1 / p)^{n}}\right), & 0 \leq x<1 .
\end{array}
$$

LEMMA 2. The functions $\bar{S}$ and $\underline{S}$ have the following properties:

(i) $\underline{S}(x) \leq \bar{S}(x)$ for $0 \leq x<1$;

(ii) $\lim _{x \rightarrow 1^{-}} \underline{S}(x)=+\infty$;

(iii) if $0 \leq x<1$ and $\beta=1 / \ln (q / p)$, then

$$
\beta I\left(x^{1-q}\right) \leq \bar{S}(x) \leq \beta I\left(x^{(1-q)(p / q)}\right) ;
$$

(iv) if $0 \leq x<1$ and $\alpha=1 / \ln (1 / p)$, then

$$
\alpha(1-\varepsilon) I\left(x^{1-p}\right) \leq \underline{S}(x) \leq \alpha I\left(x^{(1-p) p}\right) .
$$

Proof. (i) The proof follows from the monotonicity of the function $K^{-1}$ and the inequality $(1-q) q^{n} \leq 1-p$ valid for any $n \in \mathbb{N}$.

(ii) Since

$$
\underline{S}(x) \geq \sum_{n=0}^{N} K^{-1}\left((1-\varepsilon) x^{(1-p)(1 / p)^{n}}\right),
$$

we obtain

$$
\lim _{x \rightarrow 1^{-}} \underline{S}(x) \geq(N+1) K^{-1}(1-\varepsilon)
$$

for any natural $N$, proving the assertion.

(iii) First we prove the left-hand inequality of (9). Let $\Psi(x)=x^{q / p}$ for $0 \leq x<1$, and let

$$
x_{0}=x^{1-q}, \quad x_{n+1}=\Psi\left(x_{n}\right), \quad n=0,1,2, \ldots
$$

Then the sequence $x_{n}$ is decreasing and convergent to zero. Putting $a=x_{i+1}$ and $b=x_{i}$ in (6) we get

$$
\int_{x_{i+1}}^{x_{i}} K^{-1}(s) \frac{d s}{s(-\ln s)} \leq\left(-\ln \frac{p}{q}\right) K^{-1}\left(x_{i}\right)=\ln (q / p) K^{-1}\left(x^{(1-q)(q / p)^{i}}\right),
$$

for $i=0,1,2, \ldots$ Adding the above inequalities from $i=0$ to $n$ we get

$$
\int_{x_{n+1}}^{x^{1-q}} K^{-1}(s) \frac{d s}{s(-\ln s)} \leq \ln (q / p) \sum_{i=0}^{n} K^{-1}\left(x^{(1-q)(q / p)^{i}}\right) .
$$


Now, letting $n \rightarrow \infty$, we obtain

$$
\bar{S}(x)=\sum_{n=0}^{\infty} K^{-1}\left(x^{(1-q)(q / p)^{n}}\right) \geq \beta \int_{0}^{x^{1-q}} K^{-1}(s) \frac{d s}{s(-\ln s)}=\beta I\left(x^{1-q}\right),
$$

where $\beta=1 / \ln (q / p)$.

To prove the right-hand inequality of (9) we use the left inequality of (6) with $a=x_{i+1}$ and $b=x_{i}$. Thus

$$
\int_{x_{i+1}}^{x_{i}} K^{-1}(s) \frac{d s}{s(-\ln s)} \geq \ln (q / p) K^{-1}\left(x_{i+1}\right)=\ln (q / p) K^{-1}\left(x^{(1-q)(q / p)^{i+1}}\right)
$$

for $i=0,1,2, \ldots$ In much the same way as above we obtain

$$
I\left(x^{1-q}\right)=\int_{0}^{x^{1-q}} K^{-1}(s) \frac{d s}{s(-\ln s)} \geq \ln (q / p) \sum_{n=1}^{\infty} K^{-1}\left(x^{(1-q)(q / p)^{n}}\right) .
$$

Moreover, taking $a=x^{1-q}$ and $b=x^{(1-q)(p / q)}$ in (6) we have

$$
K^{-1}\left(x^{1-q}\right) \leq \beta \int_{x^{1-q}}^{x^{(1-q)(p / q)}} K^{-1}(s) \frac{d s}{s(-\ln s)} .
$$

Combining (11) and (12) we get

$$
\bar{S}(x) \leq \beta I\left(x^{1-q}\right)+K^{-1}\left(x^{1-q}\right) \leq \beta I\left(x^{(1-q)(p / q)}\right) .
$$

(iv) Let $\Phi(x)=x^{1 / p}$ for $0 \leq x<1$, and let

$$
x_{0}=x^{1-p}, \quad x_{n+1}=\Phi\left(x_{n}\right), \quad n=0,1,2, \ldots
$$

Then the sequence $x_{n}$ is strictly decreasing and convergent to zero. Putting $a=x_{i+1}$ and $b=x_{i}$ in (6) we have

$$
\int_{x_{i+1}}^{x_{i}} K^{-1}(s) \frac{d s}{s(-\ln s)} \leq \ln (1 / p) K^{-1}\left(x_{i}\right)
$$

for $i=0,1,2, \ldots$. Adding the above inequalities from $i=0$ to $n$ and then letting $n \rightarrow \infty$ we obtain

$$
I\left(x^{1-p}\right) \leq \ln (1 / p) \sum_{n=0}^{\infty} K^{-1}\left(x_{n}\right) .
$$

Since $k$ is nondecreasing, $K$ is convex and $K^{-1}$ is concave with $K^{-1}(0)=0$. Hence

$$
K^{-1}((1-\varepsilon) x) \geq(1-\varepsilon) K^{-1}(x),
$$

which allows us to write

$$
\underline{S}(x) \geq(1-\varepsilon) \sum_{n=0}^{\infty} K^{-1}\left(x_{n}\right) \geq \alpha(1-\varepsilon) I\left(x^{1-p}\right),
$$


where $\alpha=1 / \ln (1 / p)$. On the other hand, using (6) with $a=x_{i+1}$ and $b=x_{i}$ again we have

$$
\int_{x_{i+1}}^{x_{i}} K^{-1}(s) \frac{d s}{s(-\ln s)} \geq \ln (1 / p) K^{-1}\left(x_{i+1}\right)
$$

for $i=0,1,2, \ldots$. Summing up these inequalities we get

$$
I\left(x^{1-p}\right) \geq \ln (1 / p) \sum_{n=1}^{\infty} K^{-1}\left(x_{n}\right) .
$$

Since $K^{-1}$ is nondecreasing we have $K^{-1}((1-\varepsilon) x) \leq K^{-1}(x)$. Hence

$$
\underline{S}(x) \leq \sum_{n=0}^{\infty} K^{-1}\left(x_{n}\right) \leq \alpha I\left(x^{1-p}\right)+K^{-1}\left(x^{1-p}\right) .
$$

Now, using (6) with $a=x^{1-p}$ and $b=x^{(1-p) p}$ we estimate $K^{-1}\left(x^{1-p}\right)$ to obtain the right-hand inequality of (10). Thus the proof is complete.

Since $\bar{S}$ and $\underline{S}$ are strictly increasing functions on $[0,1)$, they have inverse functions.

REMARK 3. Let $\bar{S}^{-1}$ and $\underline{S}^{-1}$ denote the inverse functions to $\bar{S}$ and $\underline{S}$ respectively. Then

(i) $\bar{S}^{-1}(z)$ is defined for $0 \leq z<+\infty$ and $\lim _{z \rightarrow+\infty} \bar{S}^{-1}(z)=1$;

(ii) $\underline{S}^{-1}(z)$ is defined for $0 \leq z<+\infty$ and $\lim _{z \rightarrow+\infty} \underline{S}^{-1}(z)=1$.

3. Estimates of the nontrivial solution. For any continuous function $w: \mathbb{R}_{+} \rightarrow \mathbb{R}_{+}$we define the operator

$$
T w(x)=\int_{0}^{x} k(x-s)[w(s)]^{p} d s .
$$

The integral operator $T$ has the following monotonicity property:

REMARK 4. If $0 \leq w_{1}(x) \leq w_{2}(x)$ for $x \in \mathbb{R}_{+}$, then $T w_{1}(x) \leq T w_{2}(x)$ for $x \in \mathbb{R}_{+}$.

REMARK 5. If $w(x)$ is a strictly increasing function then integration by parts and the substitution $z=w(s)$ give

$$
T w(x)=\int_{0}^{x} K(x-s) d\left[w^{p}(s)\right]=\int_{0}^{w(x)} K\left(x-w^{-1}(z)\right) d z^{p} .
$$

Before the formulation of the main theorem we present some auxiliary lemmas.

Lemma 6. Let $w(x)=\bar{S}^{-1}(x)$. Then $T w(x) \geq w(x)$ for $0 \leq x<+\infty$. 
Proof. Since $\bar{S}^{-1}$ is strictly increasing and $\lim _{x \rightarrow+\infty} \bar{S}^{-1}(x)=1$, we have $w(x) \in[0,1)$ for $x \geq 0$. Moreover,

$$
\begin{aligned}
T w(x) & =\int_{0}^{w(x)} K\left(x-w^{-1}(s)\right) d\left(s^{p}\right) \geq \int_{0}^{w^{q / p}(x)} K\left(x-w^{-1}(s)\right) d\left(s^{p}\right) \\
& \geq K\left(x-w^{-1}\left(w^{q / p}(x)\right)\right)[w(x)]^{q}
\end{aligned}
$$

for $x \geq 0$. Since

$$
w^{-1}\left(w^{q / p}(x)\right)=\bar{S}(w(x))-K^{-1}\left(w^{1-q}(x)\right)=x-K^{-1}\left(w^{1-q}(x)\right),
$$

we get

$$
T w(x) \geq K\left(K^{-1}\left(w^{1-q}(x)\right)\right)[w(x)]^{q}=w(x)
$$

for $x \geq 0$.

Now we prove the following lemma:

Lemma 7. Let $w(x)=\underline{S}^{-1}(x)$. Then $T w(x) \leq w(x)$ for $0 \leq x \leq$ $K^{-1}(\varepsilon)$.

Proof. First we note that $w$ is strictly increasing and in view of Remark 3, $w(x) \in[0,1)$ for $x \geq 0$. Moreover, we obtain

$$
\begin{aligned}
T w(x) & =\int_{0}^{w(x)} K\left(x-w^{-1}(s)\right) d\left(s^{p}\right) \\
& =\int_{0}^{w^{1 / p}(x)} K\left(x-w^{-1}(s)\right) d\left(s^{p}\right)+\int_{w^{1 / p}(x)}^{w(x)} K\left(x-w^{-1}(s)\right) d\left(s^{p}\right) \\
& \leq K(x) w(x)+K\left(x-w^{-1}\left(w^{1 / p}(x)\right)\right)[w(x)]^{p}
\end{aligned}
$$

for $x \geq 0$. Since

$w^{-1}\left(w^{1 / p}(x)\right)=\underline{S}(w(x))-K^{-1}\left((1-\varepsilon) w^{1-p}(x)\right)=x-K^{-1}\left((1-\varepsilon) w^{1-p}(x)\right)$,

we finally get

$$
\begin{aligned}
T w(x) & \leq K(x) w(x)+K\left(K^{-1}\left((1-\varepsilon) w^{1-p}(x)\right)\right)[w(x)]^{p} \\
& =K(x) w(x)+(1-\varepsilon) w(x) \leq w(x)
\end{aligned}
$$

for $0 \leq x \leq K^{-1}(\varepsilon)$.

The main theorem of this section is the following existence result for equation (2). It can be proved in the same manner as Lemma 2.3 in [1].

THEOREM 8. There exists a nontrivial solution $u$ of equation (2). It is a strictly increasing function which satisfies the inequalities

$$
\bar{S}^{-1}(x) \leq u(x) \quad \text { for } 0 \leq x<+\infty,
$$


and

$$
u(x) \leq \underline{S}^{-1}(x) \quad \text { for } 0 \leq x \leq K^{-1}(\varepsilon) .
$$

Further, we will rather use the estimates for $u^{-1}(x)$ which follow from (14) and (15):

$$
\underline{S}(x) \leq u^{-1}(x) \leq \bar{S}(x) \quad \text { for } 0 \leq x<\delta^{*},
$$

where

$$
\begin{aligned}
& \delta^{*}=\min \left\{1, \delta_{\varepsilon}\right\}, \\
& \delta_{\varepsilon}=\sup \left\{x: u^{-1}(x) \leq K^{-1}(\varepsilon)\right\} .
\end{aligned}
$$

REMARK 9. Since the value of $\delta_{\varepsilon}$ is difficult to determine we will use its approximation. For simplification, it will be denoted in the same way. Using Lemma 2, for such an approximation one can take any positive solution of the inequality

$$
\beta I\left(\delta_{\varepsilon}^{(1-q)(p / q)}\right) \leq K^{-1}(\varepsilon) .
$$

For any $N \in \mathbb{N}$ we decompose $\bar{S}$ and $\underline{S}$ as

$$
\bar{S}(x)=\bar{S}_{N}(x)+\bar{R}_{N}(x), \quad \underline{S}(x)=\underline{S}_{N}(x)+\underline{R}_{N}(x),
$$

for $x \in\left[0, \delta^{*}\right)$, where

$$
\bar{S}_{N}(x)=\sum_{i=0}^{N} K^{-1}\left(x^{(1-q)(q / p)^{i}}\right), \quad \underline{S}_{N}(x)=\sum_{i=0}^{N} K^{-1}\left((1-\varepsilon) x^{(1-p)(1 / p)^{i}}\right),
$$

and $\bar{R}_{N}(x)$ and $\underline{R}_{N}(x)$ are the corresponding rests.

LEMMA 10. We have

$$
\beta\left(\bar{I}_{0}(x)-\bar{I}_{N+1}(x)\right) \leq \bar{S}_{N}(x) \leq \beta\left(\bar{I}_{-1}(x)-\bar{I}_{N}(x)\right)
$$

and

$$
\underline{S}_{N}(x) \geq \alpha(1-\varepsilon)\left(\underline{I}_{0}(x)-\underline{I}_{N+1}(x)\right)
$$

for $x \in\left[0, \delta^{*}\right)$, where

$$
\bar{I}_{i}(x)=I\left(x^{(1-q)(q / p)^{i}}\right), \quad \underline{I}_{i}(x)=I\left(x^{(1-p)(1 / p)^{i}}\right),
$$

and $\alpha=1 / \ln (1 / p), \beta=1 / \ln (q / p)$.

Before giving the proof of this lemma we make the following remark.

REMARK 11. In the notation of Lemma 10, inequalities (9) and (10) can be rewritten in the form

$$
\begin{gathered}
\beta \bar{I}_{0}(x) \leq \bar{S}(x) \leq \beta \bar{I}_{-1}(x), \\
\alpha(1-\varepsilon) \underline{I}_{0}(x) \leq \underline{S}(x) \leq \alpha \underline{I}_{-1}(x),
\end{gathered}
$$

for any $x \in\left[0, \delta^{*}\right)$. Moreover, we notice that

$$
\bar{R}_{N}(x)=\bar{S}\left(x^{(q / p)^{N+1}}\right), \quad \underline{R}_{N}(x)=\underline{S}\left(x^{(1 / p)^{N+1}}\right) .
$$


Proof of Lemma 10. First we prove (20). Let $x \in\left[0, \delta^{*}\right)$ and $x_{n}=$ $x^{(1-q)(q / p)^{n}}, n \in \mathbb{N}$. We note that

$$
\bar{S}_{N}(x)=\sum_{n=0}^{N} K^{-1}\left(x_{n}\right) .
$$

Now, we estimate $K^{-1}\left(x_{n}\right)$ using (6), which gives

$$
\beta \int_{x_{n+1}}^{x_{n}} K^{-1}(s) \frac{d s}{s(-\ln s)} \leq K^{-1}\left(x_{n}\right) \leq \beta \int_{x_{n}}^{x_{n-1}} K^{-1}(s) \frac{d s}{s(-\ln s)}
$$

for all $n \in \mathbb{N}$. Since

$$
\int_{x_{n+1}}^{x_{n}} K^{-1}(s) \frac{d s}{s(-\ln s)}=I\left(x_{n}\right)-I\left(x_{n+1}\right)=\bar{I}_{n}(x)-\bar{I}_{n+1}(x)
$$

for all $n \in \mathbb{N}$, the inequalities in (20) follow easily from (26)-(28).

To prove $(21)$, let $y \in\left[0, \delta^{*}\right)$ and $y_{n}=y^{(1-p)(1 / p)^{n}}, n \in \mathbb{N}$, and notice that

$$
\underline{S}_{N}(x)=\sum_{n=0}^{N} K^{-1}\left((1-\varepsilon) y_{n}\right) .
$$

We estimate $K^{-1}\left((1-\varepsilon) y_{n}\right)$ using (13) and the inequality (6), which gives

$$
K^{-1}\left((1-\varepsilon) y_{n}\right) \geq(1-\varepsilon) K^{-1}\left(y_{n}\right) \geq \alpha(1-\varepsilon) \int_{y_{n+1}}^{y_{n}} K^{-1}(s) \frac{d s}{s(-\ln s)}
$$

Since

$$
\int_{y_{n+1}}^{y_{n}} K^{-1}(s) \frac{d s}{s(-\ln s)}=\underline{I}_{n}(y)-\underline{I}_{n+1}(y)
$$

for all $n \in \mathbb{N}$, the inequality (21) follows from (29)-(31).

4. Estimates of the relative errors. In this part we present estimates of the relative errors of the approximations of $u^{-1}$ given by $\bar{S}_{N}$ and $\underline{S}_{N}$. Assume additionally that the function $K$ satisfies the following condition:

REMARK 12. The condition (32) is satisfied by smooth functions being of our particular interest, for example $K(x)=\exp \left(-1 / x^{\lambda}\right), \lambda \geq 1$, for $0<x<\delta^{*}$, and $K(x)=\exp \left(-\exp \left(1 / x^{\lambda}\right)\right), \lambda>0$, for $0<x<\min \left\{1 / e, \delta^{*}\right\}$.

LEMMA 13. If the function $K$ satisfies condition (32) and $\gamma>0$, then

$$
K^{-1}\left(x^{\gamma}\right) \leq \max \{1,1 / \gamma\} K^{-1}(x) \quad \text { for } x \in\left[0, \delta^{*}\right) .
$$


Proof. Let $\gamma \geq 1$. Since $x^{\gamma} \leq x$ for $0<x<1$, and $K$ is strictly increasing, we have $K^{-1}\left(x^{\gamma}\right) \leq K^{-1}(x)$ and (33) is satisfied.

Let $\gamma \in(0,1)$ and $F(x)=1 / \ln K(x)$. Then $F$ is nonincreasing. Let $z_{1}=K^{-1}\left(x^{\gamma}\right)$ and $z_{2}=K^{-1}(x)$. It follows from (32) that

$$
\frac{1}{\ln K\left(\gamma z_{1}\right)} \geq \gamma \frac{1}{\ln K\left(z_{1}\right)}=\frac{1}{\ln x}=\frac{1}{\ln K\left(z_{2}\right)} .
$$

Since $F$ is nonincreasing, we obtain

$$
K^{-1}\left(x^{\gamma}\right) \leq \frac{1}{\gamma} K^{-1}(x)
$$

and (33) holds near zero.

The following corollary follows from Lemma 13 immediately:

Corollary 14. If the function $K$ satisfies condition $(32)$ and $\gamma \in(0,1)$, then

$$
I\left(x^{\gamma}\right) \leq \frac{1}{\gamma} I(x) \quad \text { for } x \in\left(0, \delta^{*}\right) .
$$

Let us consider the relative errors of the approximations $\bar{S}_{N}$ and $\underline{S}_{N}$ of the function $u^{-1}$ on $\left(0, \delta^{*}\right)$, where $\delta^{*}$ is given by $(17)$ :

$$
\bar{E}_{N}(x)=\frac{\left|u^{-1}(x)-\bar{S}_{N}(x)\right|}{u^{-1}(x)}, \quad \underline{E}_{N}(x)=\frac{\left|u^{-1}(x)-\underline{S}_{N}(x)\right|}{u^{-1}(x)},
$$

for $N \in \mathbb{N}$ and $x \in\left(0, \delta^{*}\right)$.

THEOREM 15. The following pointwise estimates hold:

$$
\begin{aligned}
\bar{E}_{N}(x) & \leq \bar{e}_{N}(x) \\
= & \max \left\{\frac{\beta \bar{I}_{N}(x)}{\alpha(1-\varepsilon) \underline{I}_{0}(x)}, \frac{\beta q(1-p)}{\alpha(1-\varepsilon) p(1-q)}+\frac{(2-\varepsilon) \underline{I}_{N}(x)}{(1-\varepsilon) \underline{I}_{0}(x)}-1\right\},
\end{aligned}
$$

and

$$
\underline{E}_{N}(x) \leq \underline{e}_{N}(x)=\frac{\beta q(1-p)}{\alpha(1-\varepsilon) p(1-q)}+\frac{\beta \bar{I}_{N}(x)}{\alpha(1-\varepsilon) \underline{I}_{0}(x)}+\frac{\underline{I}_{N}(x)}{\underline{I}_{0}(x)}-1,
$$

for $N \in \mathbb{N}, \alpha=1 / \ln (1 / p), \beta=1 / \ln (q / p)$ and $x \in\left(0, \delta^{*}\right)$.

Proof. First we prove (36). Using (16) we have

$$
\underline{S}(x)=\underline{S}_{N}(x)+\underline{R}_{N}(x) \leq u^{-1}(x) \leq \bar{S}_{N}(x)+\bar{R}_{N}(x)=\bar{S}(x)
$$

for $x \in\left[0, \delta^{*}\right)$. Thus

$$
\bar{E}_{N}(x) \leq \max \left\{\frac{\bar{R}_{N}(x)}{\underline{S}(x)}, \frac{\left|\underline{S}(x)-\bar{S}_{N}(x)\right|}{\underline{S}(x)}\right\} \quad \text { for } x \in\left(0, \delta^{*}\right) .
$$

With the help of Remark 11 one can show that

$$
\frac{\bar{R}_{N}(x)}{\underline{S}(x)}=\frac{\bar{S}\left(x^{(q / p)^{N+1}}\right)}{\underline{S}(x)} \leq \frac{\beta \bar{I}_{-1}\left(x^{(q / p)^{N+1}}\right)}{\alpha(1-\varepsilon) \underline{I}_{0}(x)}=\frac{\beta \bar{I}_{N}(x)}{\alpha(1-\varepsilon) \underline{I}_{0}(x)} .
$$


On the other hand, we have

$$
\frac{\left|\underline{S}(x)-\bar{S}_{N}(x)\right|}{\underline{S}(x)} \leq \frac{\bar{S}_{N}(x)-\underline{S}_{N}(x)+\underline{R}_{N}(x)}{\underline{S}(x)}
$$

in the vicinity of zero. From Lemma 10 and Remark 11 we get

$$
\begin{aligned}
\frac{\bar{S}_{N}(x)-\underline{S}_{N}(x)}{\underline{S}(x)} & \leq \frac{\beta}{\alpha(1-\varepsilon)} \frac{\bar{I}_{-1}(x)}{\underline{I}_{0}(x)}+\frac{\underline{I}_{N+1}(x)}{\underline{I}_{0}(x)}-1 \\
& \leq \frac{\beta}{\alpha(1-\varepsilon)} \frac{I\left(x^{(1-q)(p / q)}\right)}{I\left(x^{1-p}\right)}+\frac{\underline{I}_{N}(x)}{\underline{I}_{0}(x)}-1 .
\end{aligned}
$$

Let $\gamma=\frac{p(1-q)}{q(1-p)}$. Then we note that $\gamma \in(0,1)$ for any $p, q \in(0,1)$ such that $0<p<q<1$. Using Corollary 14 we obtain

$$
I\left(x^{(1-q)(p / q)}\right) \leq \frac{1}{\gamma} I\left(x^{1-p}\right)
$$

near zero. Moreover,

$$
\frac{\underline{R}_{N}(x)}{\underline{S}(x)}=\frac{\underline{S}\left(x^{(1 / p)^{N+1}}\right)}{\underline{S}(x)} \leq \frac{\underline{I}_{-1}\left(x^{(1 / p)^{N+1}}\right)}{(1-\varepsilon) \underline{I}_{0}(x)}=\frac{\underline{I}_{N}(x)}{(1-\varepsilon) \underline{I}_{0}(x)}
$$

for any $x \in\left(0, \delta^{*}\right)$. Combining inequalities (40)-(42) we obtain

$$
\frac{\left|\underline{S}(x)-\bar{S}_{N}(x)\right|}{\underline{S}(x)} \leq \frac{\beta q(1-p)}{\alpha(1-\varepsilon) p(1-q)}+\frac{(2-\varepsilon) \underline{I}_{N}(x)}{(1-\varepsilon) \underline{I}_{0}(x)}-1 .
$$

Finally, inequality (36) follows from (39) and (43).

Now, observe that by (38) we get

$$
\underline{E}_{N}(x) \leq \frac{\bar{S}_{N}(x)-\underline{S}_{N}(x)+\bar{R}_{N}(x)}{\underline{S}(x)} \quad \text { for } x \in\left(0, \delta^{*}\right) .
$$

Therefore (37) can be proved similarly to (36).

5. Numerical examples. To illustrate the theoretical results of the previous section, we give two numerical examples that present approximations of $u^{-1}$ given by $\bar{S}_{N}$ and $\underline{S}_{N}$. All computations are made on the interval $\left(0, \delta^{*}\right]$, where $\delta^{*}$ is given by (17). In both examples we take $N=200, p=1 / 3$, $q=1 / 2$ and $\varepsilon=10^{-3}$.

EXAMPLE 5.1. We consider equation (2) with $k(x)=K^{\prime}(x)$, where $K(x)=\exp \left(-1 / x^{\lambda}\right), \lambda>0$. In this case $K^{-1}(x)=1 /(\ln (1 / x))^{1 / \lambda}$. Then the integral in (5) takes the form

$$
I(x)=\int_{0}^{x} \frac{d s}{(\ln (1 / s))^{1 / \lambda} s(-\ln s)}=\frac{\lambda}{(\ln (1 / x))^{1 / \lambda}},
$$




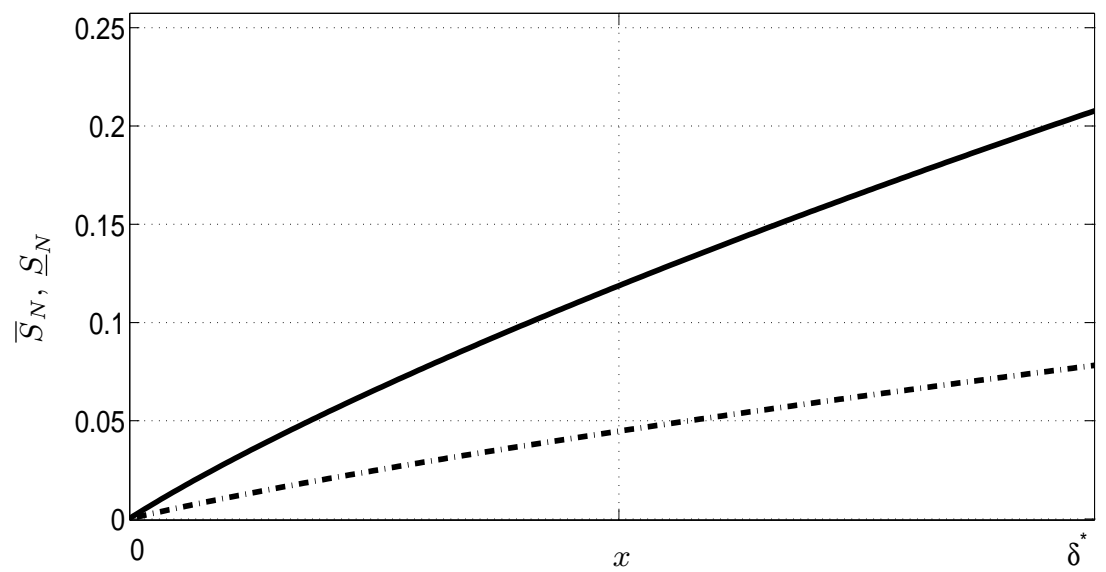

Fig. 1. Estimates of $u^{-1}$ given by $\bar{S}_{N}$ (solid line) and $\underline{S}_{N}$ (dashed line) with $N=200$, $\lambda=3 / 2, p=1 / 3, q=1 / 2$ and $\delta^{*}=9.12 \cdot 10^{-65}$

so it is convergent for $0<x<1$. This implies that equation (2) has nontrivial solutions for $\lambda>0$. If we take, for example $\lambda=3 / 2$, then using Remark 9 we find $\delta^{*}=9.12 \cdot 10^{-65}$. Then we calculate the approximations of $u^{-1}$ (Fig. 1), given by

$$
\begin{aligned}
\bar{S}_{N}(x) & =\sum_{i=0}^{N} \frac{1}{\left((1-q)(q / p)^{i} \ln (1 / x)\right)^{1 / \lambda}}, \\
\underline{S}_{N}(x) & =\sum_{i=0}^{N} \frac{1}{\left(-\ln (1-\varepsilon)+(1-p)(1 / p)^{i} \ln (1 / x)\right)^{1 / \lambda}} .
\end{aligned}
$$

Since the integral $I(x)$ is given explicitly, we easily note that $\bar{e}_{N}(x)$ and $\underline{e}_{N}(x)$ do not depend on $x$ and are given by

$$
\begin{aligned}
& \bar{e}_{N}=\frac{\beta q(1-p)}{\alpha(1-\varepsilon) p(1-q)}+\frac{2-\varepsilon}{1-\varepsilon} p^{N / \lambda}-1, \\
& \underline{e}_{N}=\frac{\beta q(1-p)}{\alpha(1-\varepsilon) p(1-q)}+\frac{\beta(1-p) p^{N}}{\alpha(1-\varepsilon)(1-q) q^{N}}+p^{N / \lambda}-1 .
\end{aligned}
$$

EXAMPLE 5.2. We consider equation (2) with $k(x)=K^{\prime}(x)$, where $K(x)=\exp \left(-\exp \left(1 / x^{\lambda}\right)\right), \lambda>0$. Then $K^{-1}(x)=1 /(\ln \ln (1 / x))^{1 / \lambda}$ and for $0<\lambda<1$ the integral $I(x)$ in (5) has the form

$$
I(x)=\int_{0}^{x} \frac{d s}{(\ln \ln (1 / s))^{1 / \lambda} s(-\ln s)}=\frac{\lambda}{1-\lambda}(\ln \ln (1 / x))^{(\lambda-1) / \lambda},
$$

for $0<x<1 / e$. Thus it is convergent and consequently (2) has a nontrivial solution. For $\lambda \geq 1$ it can be shown similarly that $I(x)$ is divergent and (2) 


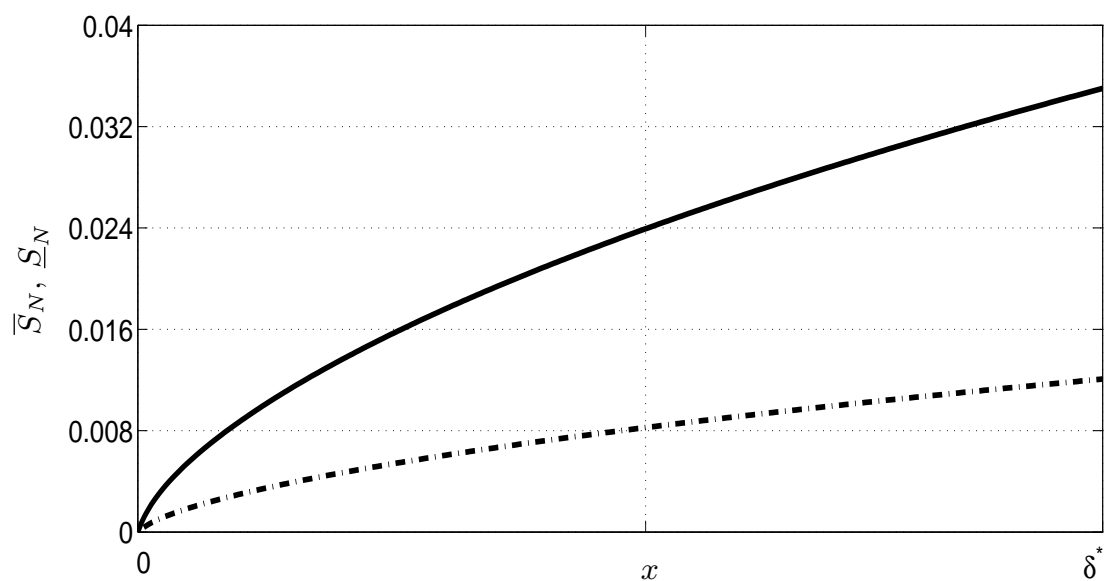

Fig. 2. Estimates of $u^{-1}$ given by $\bar{S}_{N}$ (solid line) and $\underline{S}_{N}$ (dashed line) with $N=200$, $\lambda=1 / 5, p=1 / 3, q=1 / 2$ and $\delta^{*}=1.53 \cdot 10^{-9}$

has no nontrivial solution. Taking $\lambda=1 / 5$ we calculate $\delta^{*}=1.53 \cdot 10^{-9}$.Then the approximations (Fig. 2) are given for $x \in\left(0, \delta^{*}\right]$ with

$$
\begin{aligned}
\bar{S}_{N}(x) & =\sum_{i=0}^{N} \frac{1}{(\ln \ln (1 / x)+\ln (1-q)+i \ln (q / p))^{1 / \lambda}}, \\
\underline{S}_{N}(x) & =\sum_{i=0}^{N} \frac{1}{\left(\ln \ln \left(A_{i} / x\right)+\ln (1-p)+i \ln (1 / p)\right)^{1 / \lambda}},
\end{aligned}
$$

where $A_{i}=(1-\varepsilon)^{p^{i} /(p-1)}$. Hence we get

$$
\begin{aligned}
& \bar{e}_{N}(x)=\frac{\beta q(1-p)}{\alpha(1-\varepsilon) p(1-q)}+\frac{2-\varepsilon}{1-\varepsilon} \underline{\eta}_{N}(x)-1, \\
& \underline{e}_{N}(x)=\frac{\beta q(1-p)}{\alpha(1-\varepsilon) p(1-q)}+\frac{\beta \bar{\eta}_{N}(x)}{\alpha(1-\varepsilon)}+\underline{\eta}_{N}(x)-1,
\end{aligned}
$$

where

$$
\begin{aligned}
& \bar{\eta}_{N}(x)=\left(\frac{\ln (1-p)+\ln \ln (1 / x)}{\ln (1-q)+N \ln (q / p)+\ln \ln (1 / x)}\right)^{(1-\lambda) / \lambda}, \\
& \underline{\eta}_{N}(x)=\left(\frac{\ln (1-p)+\ln \ln (1 / x)}{\ln (1-p)+N \ln (1 / p)+\ln \ln (1 / x)}\right)^{(1-\lambda) / \lambda} .
\end{aligned}
$$


Since the decreasing functions $\bar{\eta}_{N}(x), \eta_{N}(x)$ tend to 1 as $x \rightarrow 0^{+}$, we note that in this case we have uniform estimates of the relative errors given by

$$
\begin{aligned}
& \bar{e}_{N}=\frac{\beta q(1-p)}{\alpha(1-\varepsilon) p(1-q)}+\frac{2-\varepsilon}{1-\varepsilon}-1, \\
& \underline{e}_{N}=\frac{\beta q(1-p)}{\alpha(1-\varepsilon) p(1-q)}+\frac{\beta}{\alpha(1-\varepsilon)} .
\end{aligned}
$$

Acknowledgements. The author thanks the referee for many helpful comments and suggestions, which led to a considerably improved version of the paper.

\section{References}

[1] P. J. Bushell and W. Okrasiński, Uniqueness of solutions for a class of nonlinear Volterra integral equations with convolution kernel, Math. Proc. Cambridge Philos. Soc. 106 (1989), 547-552.

[2] - - - Nonlinear Volterra integral equations with convolution kernels, J. London Math. Soc. 41 (1990), 503-510.

[3] N. Karapetiants, A. Kilbas, M. Saigo and S. Samko, Upper and lower bounds for solutions of nonlinear Volterra convolution integral equations with power nonlinearity, J. Integral Equations Appl. 12 (2002), 421-448.

[4] W. Mydlarczyk, The existence of nontrivial solutions of Volterra equations, Math. Scand. 68 (1991), 83-88.

[5] -, A Volterra inequality with the power type nonlinear kernel, J. Inequal. Appl. 6 (2001), 625-631.

[6] W. Okrasiński, Nontrivial solutions for a class of nonlinear Volterra equations with convolution kernel, J. Integral Equations Appl. 3 (1991), 399-409.

[7] —, Nontrivial solutions to nonlinear Volterra integral equations, SIAM J. Math. Anal. 22 (1991), 1007-1015.

Faculty of Mathematics, Computer Science and Econometrics

University of Zielona Góra

Podgórna 50

65-246 Zielona Góra, Poland

E-mail: M.Niedziela@wmie.uz.zgora.pl

Received on 21.5.2005;

revised version on 19.6.2008 
